\title{
A reliable data transfer protocol based on twin paths and network coding for underwater acoustic sensor network
}

\author{
Shaobin Cai ${ }^{*}$, Nianmin $\mathrm{Yao}^{2}$ and Zhenguo $\mathrm{Gao}^{2}$
}

\begin{abstract}
MPNC (multiple path and network coding) is proposed as a reliable transport protocol for UWASN (underwater acoustic sensor network). In MPNC, after three disjoint paths being established, two groups of packets ( $A$ and $B$ ), coded by network coding, are transmitted over the two side paths respectively, and another group of packets $C=A \oplus B$ are transmitted over the middle path. That is, any two paths can work together as a redundant path for another path, and the reliable transmission of one packet can be guaranteed by 1.5 packets. In order to reduce the number of redundant packets without affecting the transmission reliability, the packets of group $C$ are coded further at special ratio by network coding as shareable redundant packets according to the link error, and a reliable transport protocol TPNC (twin path and network coding) based on two paths and network coding is proposed in this paper. In TPNC, after two disjoint paths, called twin paths, being established, two groups of packets ( $A$ and $B$ ), coded by network coding, are transmitted with their own shareable redundant packets over the two paths respectively to guarantee the data packet transmission reliability. The results of simulations show that, compared with MPNC, TPNC can acquire similar data delivery ratio with lower energy consumption.
\end{abstract}

Keywords: Underwater acoustic sensor network; Transport protocol; Reliability; Twin paths; Network coding

\section{Introduction}

UWASN (underwater acoustic sensor network) is a special kind of WSN (wireless sensor network), which is consisted of underwater acoustic sensor nodes. The UWASN can be deployed for real-time warship monitoring, oceanographic data collection, environmental monitoring, and disaster prevention, etc. Hence, lots of researches have been done on it [1-3].

The design of a reliable data transfer protocol for UWASN is challenging due to the specific characteristics of acoustic channels: high bit error rates, high energy consumption, limited available bandwidth, low transmission speed, and long unstable packet delivery delay.

Traditionally, the data transmission reliability is guaranteed by acknowledgments and/or FEC (forward error correction). However, the data transmission protocols based on traditional acknowledgments, for example, ARQ, are

\footnotetext{
* Correspondence: caishaobin@hrbeu.edu.cn

${ }^{1}$ Computer Science Department, Harbin Engineering University, Harbin 150001, China

Full list of author information is available at the end of the article
}

not suitable for UWASN [4-6] because their acknowledgment packets not only prolong the transmission delay but also waste lots of limited bandwidth. Therefore, to reduce the bandwidth waste and transmission delay, caused by acknowledgment packets, without reducing data transmission reliability, some improved ARQ-based protocols [6-9] use data packets as implicit acknowledgments; some protocols [10-14], such as segmented data reliable transport (SDRT) [12,13], NCRF (network coding [14] in rateless fashion) [15], and NCIA (network coding with implicit acknowledgment) [15], send coded packets in burst, which only needs a acknowledgment packet; some protocols, such as ADELIN (adaptive reliable transport) [16,17], VBF-NC (vector-based forwarding-network coding) [18] and MPNC (multiple path and network coding) [19], guarantee transmission reliability only by sending enough coed packets.

In MPNC, three disjoint paths are established firstly, and then two groups of packets $(A$ and $B)$, coded by network coding, are transmitted over the two side paths individually; another group of coded packets $(C=A \oplus B)$ 
are transmitted over the middle path (Figure 1). So, any two paths can work together as a redundant path for another path. That is, the reliable transmission of one packet can be guaranteed by 1.5 packets in MPNC.

In order to reduce data transmission overhead, the packets of group $C$ are coded further at special ratio by network coding as shareable redundant packet according to the link error, and TPNC (twin path and network coding) is proposed in this paper. In TPNC, two disjoint paths are established firstly, and then, one group of packets $A(B)$, coded by network coding, are transmitted with its respective shareable redundant packets on one path.

The rest of the paper is arranged as the following: firstly, TPNC is proposed in chapter 2; secondly, the performances of TPNC are analyzed in chapter 3; finally, a conclusion is drawn in chapter 4 .

\section{TPNC}

Figure 2 discribes how TPNC works. In Figure 2, the numbers $\left(7_{i}+0,7_{i}+1,7_{i}+2, \ldots, 7_{i}+4 n\right)$ are the sequence numbers of time slices, at which the nodes send out their coded packets as following:

(1) $O_{\mathrm{A}}$ and $O_{\mathrm{B}}$ are two groups of $K$ original packets. They are coded by network coding individually to form two coded packet groups $(A$ and $B$ ), each of which has $K+K_{1}$ coded packets.

(2) According to the distance between node $S$ and $A_{1}$ $\left(B_{1}\right)$, node $S$ calculates the link error ratio $e_{a 1}\left(e_{b 1}\right)$; node $S$ coded every $\lceil 1 / e]\left(e=\max \left(e_{a 1}, e_{b 1}\right)\right.$ packets of group $C(C=A \oplus B)$ to form a packet of group $D$ by network coding.

(3) Node $S$ codes packets of group $D$ by network coding to acquire the $D(1+\lfloor e\rfloor)$ packets and forms packet group $E$.

(4) At time slice 0 , node $S$ sends out the packets of group $A$ with the packets of group $E_{1}$ (the first half of group $E$ ) to guarantee the packet transmission reliability.
(5) At time slice 1 , node $S$, it sends out the packets of group $B$ with the packets of group $E_{2}$ (the latter half of packet group $E$ ) to guarantee the packet transmission reliability.

(6) At time slice 2, node $A_{1}$ broadcasts its received packets of group $A$ and group $E_{1}$ to improve the packet transmission reliability.

(7) At time slice 3 , node $B_{1}$ broadcasts its received packets of group $B$ and group $E_{2}$ to improve the packet transmission reliability.

(8) By their received packets of group $A$, group $B$, and group $E$ from node $S$, node $A_{1}$, and node $B_{1}$, node $A_{1}$ and node $B_{1}$ can decode these packets to acquire the original packets of group $O_{\mathrm{A}}$ and group $O_{\mathrm{B}}$.

(9) At time slice 4, according to link error ratio, calculated from the distance between node $A_{1}$ and node $A_{2}$, node $A_{1}$ forms packet group $E$ and sends out the packets of group $A$ and group $E_{1}$, which are not sent out at time slice 2 .

(10) At time slice 5, according to link error ratio, calculated from the distance between node $B_{1}$ and node $B_{2}$, node $B_{1}$ forms packet group $E$ and sends out the packets of group $B$ and group $E_{2}$, which are not sent out at time slice 3 .

(11) At time slice 6, node $A_{2}$ broadcasts its received packets of group $A$ and group $E_{1}$ to improve transmission reliability.

(12) At time slice 7, node $B_{2}$ broadcasts its received packets of group $B$ and group $E_{2}$ to improve transmission reliability.

(13) By their received packets of group $A$, group $B$, and group $E$ from node $A_{1}$, node $A_{2}$, node $B_{1}$, and node $B_{2}$, node $A_{2}$ and node $B_{2}$ can acquire the original packets of group $O_{\mathrm{A}}$ and group $O_{\mathrm{B}}$.

(14) Node $A_{2}$ and node $B_{2}$ do as node $A_{1}$ and node $B_{1}$ do.

By the way above, packets of group $A, B$, and $E$ are transmitted over the twin path, and node $E$ can acquire the original packets of group $O_{\mathrm{A}}$ and group $O_{\mathrm{B}}$

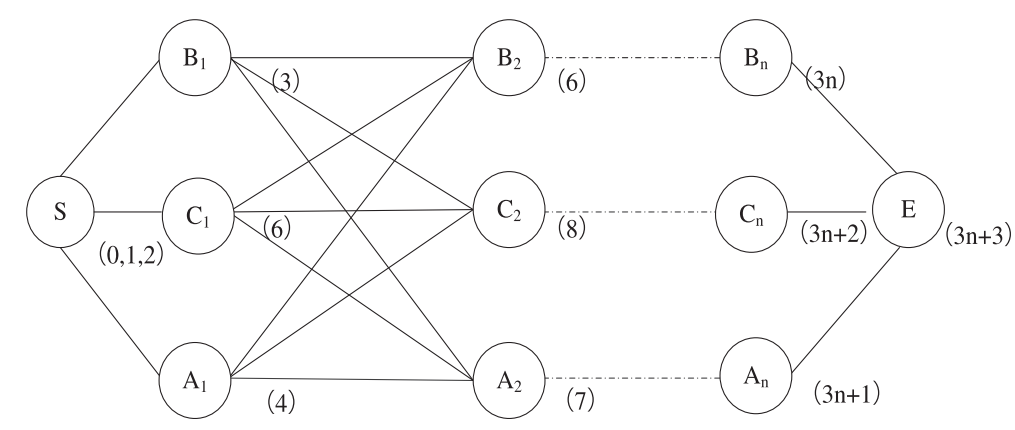

Figure 1 MPNC work flows. 


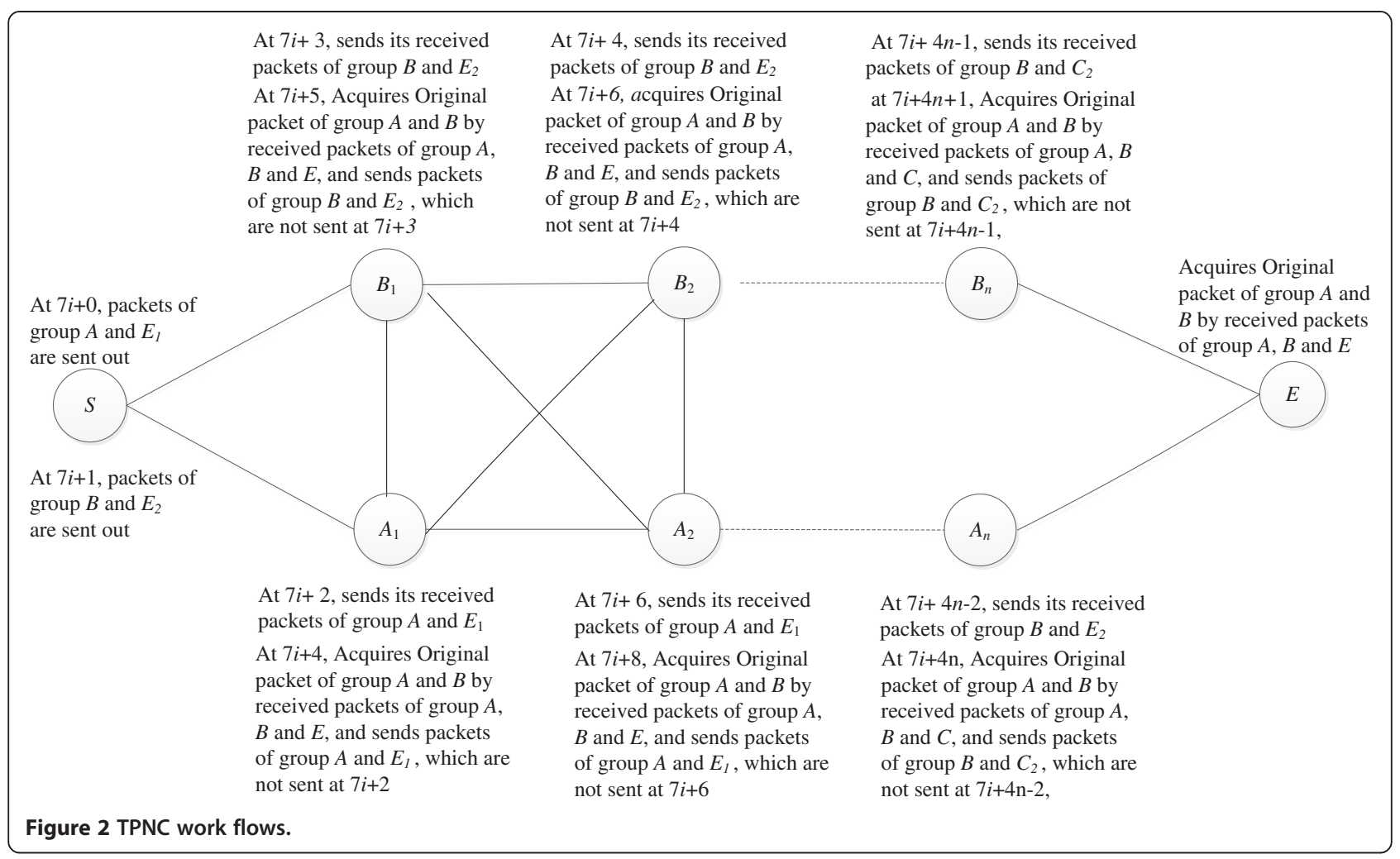

by decoding its received coded packets of group $A, B$, and $E$ finally.

From the descriptions above, it can be known that the packet number of group $E$ is much less than that of group C. So, the data transmission overhead of TPNC should be much less than that of MPNC.

\section{Performance analysis}

In this section, the performance of protocols is analyzed in a 9-hop UWASN. The simulation environment of MATLAB and Aqua-sim [20] is defined according to Zheng's work [18]. In the experiments, the bit error of channel is a direct ratio to the distance between nodes when the optimal frequency $f(d)$ is used for their communication [21]. Furthermore, in order to acquire average results, every experiment is done 1000 times.

In the following experiments, the length of data packet is 50 bytes; the number of redundant packets $K^{\prime}$ for a 15-packet group is defined as the following [19]:

(1) $K^{\prime}=0$, when $d=<10 \mathrm{~km}$;

(2) $K^{\prime}=5$, when $10 \mathrm{~km}<d=<30 \mathrm{~km}$;

(3) $K^{\prime}=10$, when $30 \mathrm{~km}<d=<35 \mathrm{~km}$;

(4) $K^{\prime}=15$, when $35 \mathrm{~km}<d=<37.5 \mathrm{~km}$;

(5) $K^{\prime}=18$, when $37.5 \mathrm{~km}<d<41.5 \mathrm{~km}$.

The successful delivery ratio and the normalized energy consumption are defined in this paper to describe the performance of protocol. The normalized energy consumption is defined as the following:

$$
T=\frac{\sum_{i=1}^{H} T_{i}}{R}
$$

In the above formula, $R$ presents the successful delivery ratio; $T_{\mathrm{i}}$ presents the number of average received packets of a node.

\subsection{MATLAB analysis}

MATLAB is used to analyze the performance of the protocols firstly in this sub-section.

Figure 3 describes the relationship between the successful delivery ratio and the node distance. From Figure 3, it can be known that TPNC is as strong as MPNC and is stronger than VBF-NC. That is, the successful delivery ratios of TPNC, MPNC, and VBF-NC are all high enough to guarantee transmission reliability when the distance between nodes is less than $35 \mathrm{~km}$. However, the delivery ratio of VBF-NC decreases greatly because its redundant packets transmitted on the forwarding set are not enough to guarantee data transmission reliability; the delivery ratios of both MPNC and TPNC only vibrate lightly because more redundant packets are used with the increasing of distance between nodes by stages, when the distance between nodes is more than $35 \mathrm{~km}$. 


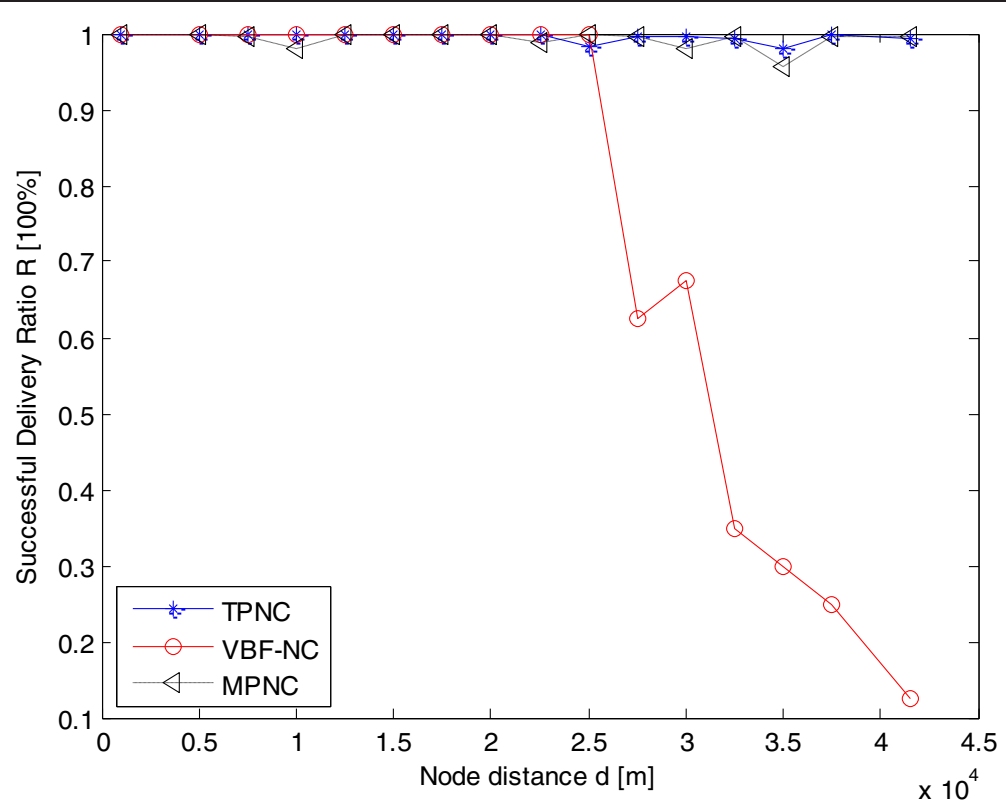

Figure 3 The relationship between successful delivery ratio and node distance.

Figure 4 describes the relationship between the normalized energy consumption and the node distance. From Figure 4, it can be seen that the normalized energy consumption of TPNC is much lower than those of MPNC and VBF-NC because fewer redundant packets are used by TPNC to guarantee the transmission reliability when the distance between nodes is small. With the increasing of the distance between nodes, more and more redundant packets are used by TPNC and MPNC to maintain their successful delivery ratio. So, the normalized energy consumptions of TPNC and MPNC increase with the increasing of the redundant packets. However, compared with MPNC, fewer redundant packets are used by TPNC, and less energy is consumed by TPNC.

From Figures 3 and 4, a conclusion can be drawn that the data delivery ratios of TPNC and MPNC are similar, and the normalized energy consumption of TPNC is much lower than that of MPNC because TPNC can

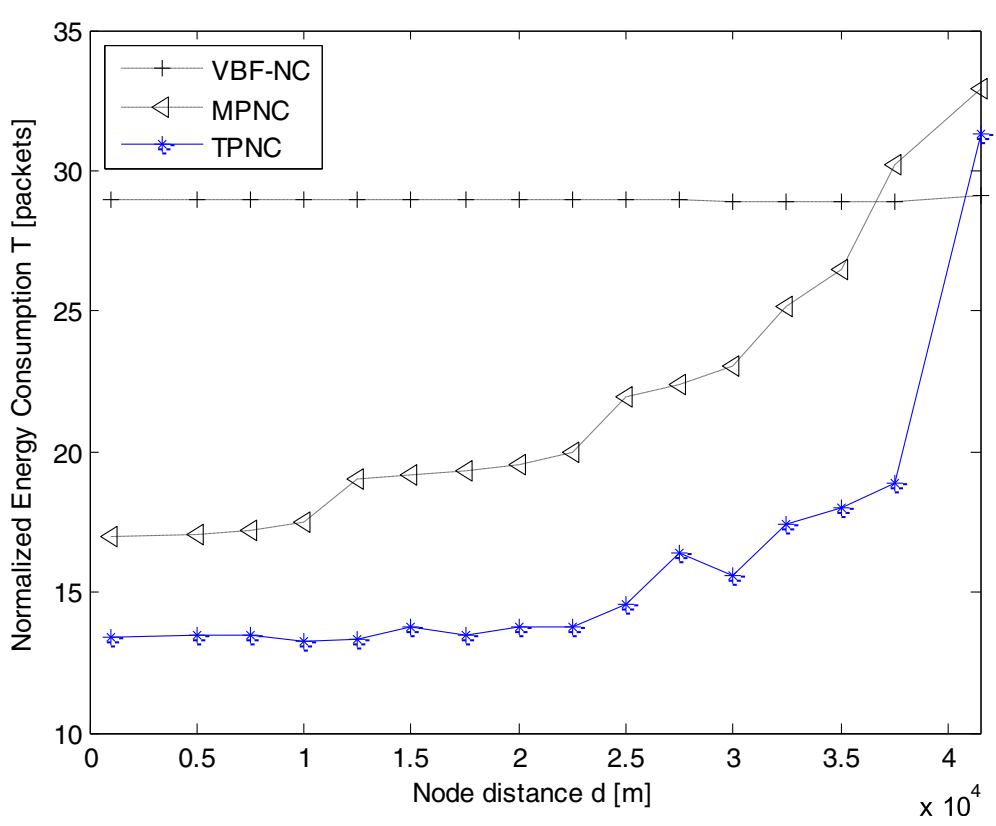

Figure 4 The relationship between normalized energy consumption and node distance. 


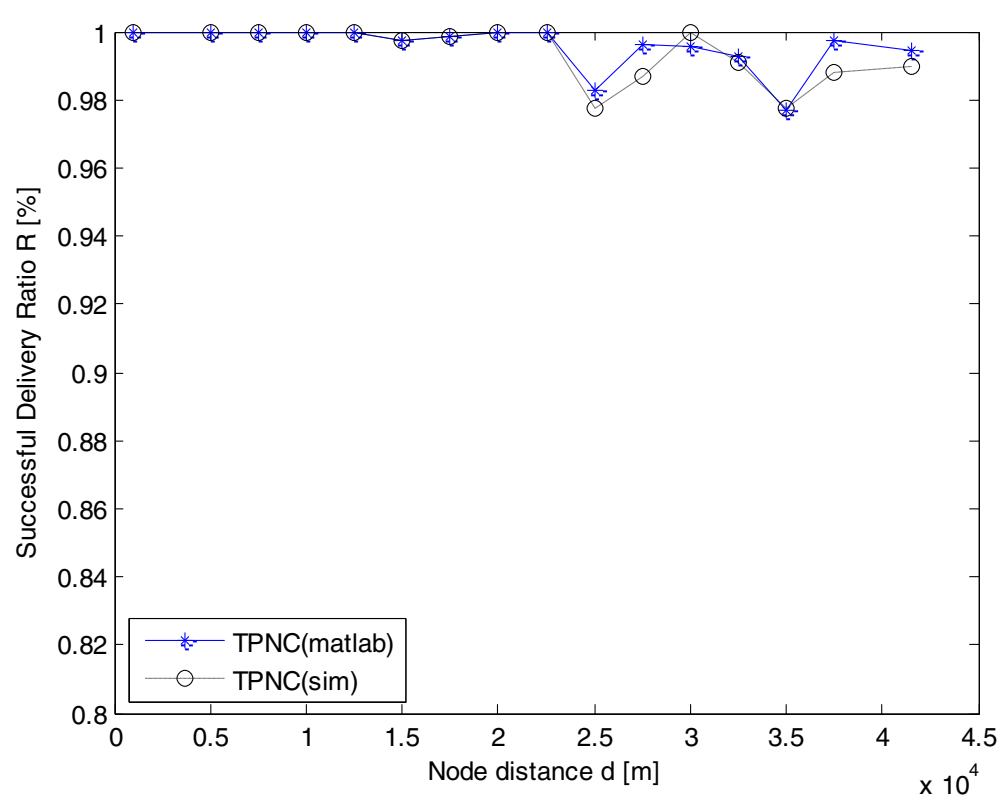

Figure $\mathbf{5}$ The relationship between successful delivery ratio and node distance.

guarantee its data transmission reliability by less redundant packets.

\subsection{Analysis by Aqua-sim}

In order to approve the analysis results of MATLAB above, the performance of the protocols is analyzed by Aqua-sim [20], which is specially developed for UWASN on the basis of NS-2 by Underwater Wireless Sensor Network Lab of Connecticut University, in this sub-section.
In Figures 5 and 6, the comparison between analysis results of MATLAB and simulation results of Aqua-sim is done. In these figures, 'Matlab' presents the analysis results of MATLAB; 'Sim' presents the simulation results of Aqua-sim. From Figures 5 and 6, it is shown that the successful delivery ratio and normalized energy consumption of Matlab and Sim are similar. There are only a few differences, which should be caused by the noise of environment during the simulation of Aqua-sim. So,

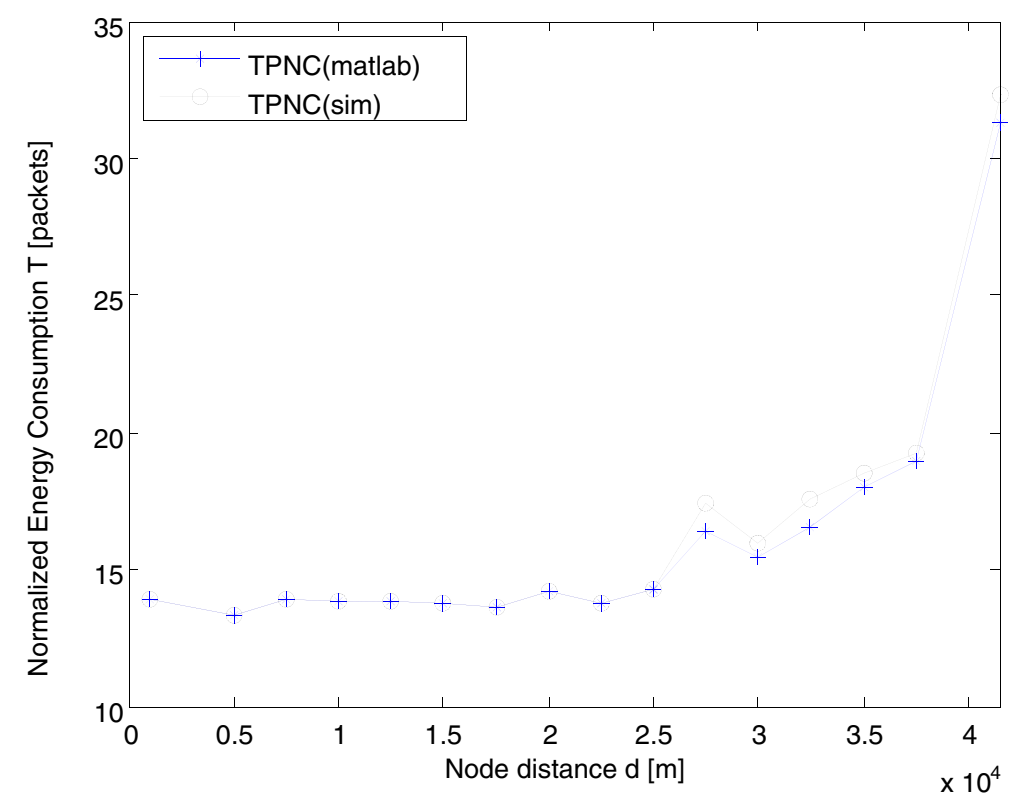

Figure 6 The relationship between normalized energy consumption and node distance. 
the simulation results can prove that the Matlab analysis results are right.

\section{Conclusion}

On the basis of analyzing the existed reliable data transfer protocols for UWASN, TPNC is proposed to improve MPNC in this paper. In order to reduce the number of redundant packets, which belong to the group $C$ in MPNC, without affecting the transmission reliability, the packets of the group $C$ are coded further by network coding as shareable redundant packet according to the link error. And then, two groups of packets $(A$ and $B)$, coded by network coding, are transmitted with their respective shareable redundant packets over the two established disjoint paths to transfer the data packet reliably. The simulation results show that the redundant packet number of TPNC is reduced greatly without affecting the strength of TPNC protocol. So, compared with MPNC, TPNC can acquire similar data delivery ratio with lower energy consumption.

\section{Competing interests}

The authors declare that they have no competing interests.

\section{Acknowledgments}

The paper is supported by National Science foundation of China (41176082, 61073082), supported by Program for New Century Excellent Talents in University (NCET-13-0753), Specialized Research Fund for the Doctoral Program of Higher Education of China (20132304110031), National Science foundation of Heilongjiang Province, China (42400621-1-14076), and Specialized Research Fund for the Innovation talents of science and technology (2014RFQXJ012).

\section{Author details}

${ }^{1}$ Computer Science Department, Harbin Engineering University, Harbin 150001, China. ${ }^{2}$ Computer Science Department, Dalian University of Technology, Dalian 116024, China.

Received: 3 July 2014 Accepted: 19 January 2015

Published online: 14 February 2015

\section{References}

1. IF Akyildiz, D Pompili, T Melodia, Underwater acoustic sensor networks: research challenges[]]. Ad Hoc Netw. 3(3), 257-279 (2005)

2. JO'Donnell, D Codiga, C Edwards, D Ullman, D Hebert, J Rice, E Levine, P Steggman, I Babb. Front resolving observational network with telemetry (FRONT) [R]. Connecticut university groton marine science inst. 2002

3. About AOSN II and Adaptive Sampling. http://www.princeton.edu/ dcsl/ aosn/

4. M Gao, W-S Soh, M Tao, A transmission Scheme for continuous ARQ protocols over underwater acoustic channels. ICC'09. 1-5 (2009)

5. J W Lee, J Y Cheon, H-S Cho, A cooperative ARQ scheme in underwater acoustic sensor networks. OCEANS'10. 1-5 (2010)

6. H-P Tan, WKG Seah, L Doyle, A multi-hop ARQ protocol for underwater acoustic networks. OCEANS'07. 1-6 (2007)

7. Valera A, Pius W, Q. Lee, H-P Tan, H Liang, WKG Seah, Implementation and evaluation of multihop ARQ for reliable communications in underwater acoustic networks. OCEANS '09. 1-6 (2009)

8. J-W Lee, J-P Kim, J-H Lee, J Y Seon, KC Dho, K Son, H-S Cho, An improved ARQ scheme in underwater acoustic sensor networks. OCEANS'08. 414-432 (2008)

9. H Zhuang, H Tan, V Alvin, Z Bai, Opportunistic ARQ with bidirectional overhearing for reliable multihop underwater networking, Oceans'10. $1-6(2010)$
10. $R$ Cao, $L$ Yang, Reliable transport and storage protocol with fountain codes for underwater acoustic sensor networks. WUWNet '10. 18-21 (2010)

11. G Lv, Z Guo, H Qu, Z Che, Study of RFEC-based transport protocol in underwater acoustic sensor networks. WiCOM '08. 1-4 (2008)

12. X Peng, C JunHong, An fec-based reliable data transport protocol for underwater sensor networks. Comput. Commun. Netw. 747-753 (2007)

13. P Xie, Z Zhou, Z Peng, J-H Cui, Z Shi, SDRT: a reliable data transport protocol for underwater sensor networks. Ad Hoc Netw. 8(7), 708-722 (2010)

14. DE Lucani, M Médard, M Stojanovic, Network coding protocols for underwater networks: the benefits of implicit acknowledgement. WuWNet '07. 25-32 (2007)

15. A Rudolf, C Ning, L ShuoYen, Y Raymond, Wai-Ho, Network information flow. IEEE Trans. Inf. Theory 46(4), 1204-1216 (2000)

16. B Liu, F Garcin, F Ren, C Lin, A study of forward error correction protocols for reliable transport in underwater sensor networks. SECON'08. 197-205 (2008)

17. L Bin, C Hongyang, L Xianfu, R Fengyuan, S Kaoru, Internode distance-based redundancy reliable transport in underwater sensor networks. EURASIP J. Wirel. Commun. Netw. 1-16 (2010)

18. Z Guo, B Wang, P Xie, W Zeng, JH Cui, Efficient error recovery with network coding in underwater sensor networks. Ad Hoc Netw. 7(4), 791-802 (2009)

19. C Shaobin, G Zhenguo, Y Nianmin, A network coding based protocol for reliable data transfer in underwater acoustic sensor. Ad Hoc Netw. 11(5), 1603-1609 (2011)

20. Aqua-Sim, An NS2 based Underwater Sensor Network Simulator, Underwater Sensor Network (UWSN) Lab, University of Connecticut, URL: http://uwsn.engr.uconn.edu/ aquasim.tar.gz

21. B Liu, H Wen, F Ren, C Lin, Performance analysis of reliable transport protocols joint with the optimal frequency and optimal packet length in underwater sensor networks. WoWMoM '07. 1-5 (2008)

\section{Submit your manuscript to a SpringerOpen ${ }^{\circ}$ journal and benefit from:}

- Convenient online submission

- Rigorous peer review

- Immediate publication on acceptance

- Open access: articles freely available online

- High visibility within the field

- Retaining the copyright to your article

Submit your next manuscript at $\gg$ springeropen.com 